\title{
Long-Term Analysis of Ab-2 (Clone SN3b) Immunoreactivity as a Prognostic Factor in Breast Carcinoma
}

\author{
Klaus-Jürgen Winzer $^{a}$ Joachim Bellach ${ }^{b}$ Glen Kristiansen ${ }^{c}$

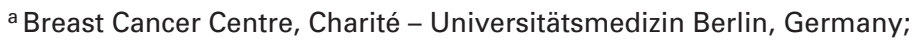 \\ ${ }^{b}$ Comprehensive Cancer Centre Charité, Charité - Universitätsmedizin Berlin, Germany; \\ ${ }^{c}$ Institute of Pathology, Rheinische Friedrich-Wilhelms-Universität Bonn, Germany
}

\section{Keywords}

Breast cancer - Prognostic factor - Ab-2 (clone SN3b) .

\section{CD24, Survival}

\section{Summary}

Background: CD24 expression has been described as a significant prognostic factor in multiple solid tumours. Most of these studies have, however, been undertaken using the Ab-2 antibody (clone SN3b), which detects a CD24-associated carbohydrate, and not the CD24 protein itself. Although its biological identity remains unclear, its prognostic significance means that detection of this carbohydrate may, nonetheless, be clinically relevant. Methods: 133 breast carcinomas were selected (pT1-2 pN0-2 M0, no secondary carcinoma, no contralateral carcinoma) from a previous SN3b expression study on a larger cohort of breast carcinomas. After updating data on follow-up observations, we carried out univariate and multivariate analysis of the prognostic significance of SN3b for total and breast cancer-specific survival. Results: A statistically significant correlation between cytoplasmic SN3b immunoreactivity and positive node status was found. Cytoplasmic SN3b also has node status-independent prognostic significance. Total survival exhibits a statistically significant dependency on cytoplasmic SN3b even for pNO cases. Conclusion: The independent prognostic value of CD24 as detected by Ab-2/ clone SN3b could replace the diagnostic axillary dissection in breast carcinoma patients if this was confirmed in further studies. Also, clarifying the exact epitope of this interesting antibody is more than warranted.

\section{Introduction}

In 2003, our group established that overexpression of CD24 in invasive breast carcinoma is prognostically unfavourable [1]. Our initial study used the monoclonal CD24 antibody Ab-2 (clone SN3b, Neomarkers (Fremont, CA, USA)), which was technically very robust and subsequently employed by innumerable groups to detect CD24, even though its precise epitope was - and still is elusive. In a later study [2], we compared the CD24 immunoreactivity patterns of clone SN3b with those of clone SWA11, which have been well characterised as specific for the CD24 protein core. This comparison revealed only a partial overlap between both antibodies. It is probable that clone SN3b detects sialic acid on Olinked glycans, which can also occur independently of the CD24 protein backbone. This implies that $\mathrm{mAb}$ SN3b is not strictly a CD24-specific antibody, which explains discrepancies with other studies, although it presumably accounts for the major component of 'CD24' immunoreactivity. This is even more of interest since SWA11 failed to show a statistically significant prognostic value, although a weak positive trend was discernible.

Our original study published in 2003 had several minor shortcomings. First, it encompassed patients from different clinics who had been diagnosed at the pathology institute of the Charité; secondly, it was based on patient data covering an average follow-up observation period of only 65 months (5-129 months); and thirdly, it lacked access to additional information on secondary tumours, etc. The acquisition of markedly prolonged follow-up data and additional patient information prompted us to reanalyse the original CD24/SN3b expression data of the patients from a single centre (Breast Cancer Centre Charité).

\section{KARGER \\ Fax +497614520714

\section{() 2015 S. Karger GmbH, Freiburg}

$1661-3791 / 15 / 0104-0273 \$ 39.50 / 0$ 


\section{Patients and Methods}

\section{Patients}

From our previous study, we identified 139 cases with primary breast carcinoma. These had undergone surgery in the surgical unit on the central Charité campus and were diagnosed at the Institute of Pathology of the Charité. 2 of these cases had other previous or concurrent malignancies and were therefore excluded from the analysis. 5 patients who subsequently developed other malignant tumours remained in the analysis. A further 4 patients who had previously been diagnosed with a contralateral breast carcinoma prior to the breast carcinoma under investigation were also excluded from the study. 4 patients who developed a simultaneous (i.e. within 6 months) contralateral breast carcinoma and 7 patients who subsequently developed a contralateral breast carcinoma were not excluded from the study. In total, 133 cases remained in the analysis. Of these, 127 cases were M0 and 6 cases were M1.

\section{Follow-Up Data}

Total survival up to a cut-off date of 30.06 .2007 was analysed. The death rates of a previously published analysis of all patients between 1984 and 1998 were underestimated since they were exclusively based on follow-up data from a single clinical tumour register that contained data established solely on further consultations with patients. These data were, therefore, supplemented with data on survival times from the Joint Cancer Register of the States of Berlin, Brandenburg, Mecklenburg-West Pomerania, Saxony-Anhalt and the Free States of Saxony and Thuringia (GKR) and from the Berlin Resident Registration Office [3].

Since no data on continued survival were available from the resident registration offices in the other German states, in particular those in the former East Germany, the number of deaths of patients from these states was weighted more heavily despite the identical recording period. This is because data from other resident registration offices, if they had been available, would have supplemented data from the clinical register prior to the cut-off date for the analysis for those patients who were no longer attending hospital consultations.

\section{Immunohistochemistry}

CD24 immunostaining was conducted as described previously [1]. Briefly, freshly cut paraffin sections were dewaxed with xylene and gradually hydrated. Antigen retrieval was achieved by pressure cooking in $0.01 \mathrm{M}$ citrate buffer for $5 \mathrm{~min}$. The primary CD24 antibody (clone SN3b; Neomarkers, Fremont, USA) was diluted 1:100 and incubated at room temperature for $2 \mathrm{~h}$. The conventional labelled streptavidin-biotin method was used for detection with alkaline phosphatase as the reporting enzyme and chromogenic development with Fast-Red. Finally, the slides were briefly counterstained and mounted.

In the initial study we kept our scoring system of the CD24 staining as simple as possible to enhance the reproducibility of our findings in future studies [1]. We evaluated the membranous and the cytoplasmic staining intensity of CD24 separately and scored the staining intensity as negative (0), or weakly $(1+)$, moderately $(2+)$ or strongly $(3+)$ positive.
Table 1. Association of CD24 (clone SN3b) with clinicopathological parameters

\begin{tabular}{lrrrrl}
\hline Characteristic & \multicolumn{2}{c}{ CD24/SN3b cytoplasmic } & \multirow{2}{*}{$\begin{array}{l}\text { Approx. } \\
\text { significance }\end{array}$} \\
\cline { 2 - 4 } & 0 & 1 & 2 & 3 & \\
\hline pT & 22 & 30 & 24 & 10 & \\
pT1 & 6 & 17 & 12 & 7 & 0.256 \\
pT2 & 1 & 0 & 0 & 1 & \\
pT3 & 1 & 0 & 2 & 0 & \\
pT4 & & & & & \\
pN & 21 & 21 & 15 & 7 & \\
pN0 & 8 & 21 & 20 & 8 & 0.013 \\
pN1 & 1 & 5 & 3 & 3 & \\
pN2 & & & & & \\
Histological grade & 13 & 14 & 10 & 5 & \\
G1 & 14 & 21 & 20 & 10 & 0.366 \\
G2 & 3 & 10 & 5 & 2 & \\
G3 & & & & & \\
Oestrogen receptor & 5 & 18 & 11 & 8 & 0.126 \\
Negative & 24 & 28 & 27 & 9 & \\
Positive & & & & & \\
\hline
\end{tabular}

${ }^{a}$ Ordinal by ordinal gamma

Table 2. Univariate Cox regression for single prognostic parameters (overall survival)

\begin{tabular}{|c|c|c|c|c|c|}
\hline \multirow{2}{*}{$\begin{array}{l}\text { Prognostic } \\
\text { parameter }\end{array}$} & \multirow{2}{*}{$\begin{array}{l}\text { Statistical } \\
\text { significance }\end{array}$} & \multicolumn{3}{|c|}{ For the analysis: } & \multirow[t]{2}{*}{$\operatorname{Exp}(B)$} \\
\hline & & $\begin{array}{l}\text { Cases } \\
\text { with } \\
\text { missing } \\
\text { data }\end{array}$ & $\begin{array}{l}\text { Censored } \\
\text { cases } \\
\text { before the } \\
\text { earliest } \\
\text { event in a } \\
\text { stratum }\end{array}$ & $\begin{array}{l}\text { Available } \\
\text { deaths }\end{array}$ & \\
\hline pT & 0.007 & 0 & 3 & 40 & 1.569 \\
\hline $\mathrm{pN}$ & 0.002 & 0 & 3 & 39 & 2.208 \\
\hline G & 0.014 & 6 & 3 & 37 & 1.765 \\
\hline ER & 0.082 & 3 & 3 & 38 & 0.566 \\
\hline $\mathrm{CD} 24 / \mathrm{SN} 3 \mathrm{~b}$ & 0.003 & 0 & 3 & 40 & 1.661 \\
\hline
\end{tabular}

$\mathrm{ER}=$ oestrogen receptor, $\operatorname{Exp}(\mathrm{B})=$ hazard ratio

\section{Results}

The mean observation period was 96.1 months (minimum 1 month, maximum 192 months). Follow-up data received from the clinical cancer registry were supplemented with data from Berlin Resident Registration Office records and the cancer registry of the new federal states of Germany (GKR). In total, 40 deaths were recorded. Because the first event (death) occurred after 6 months, 2 cases for which the follow-up period was only 1 month and 1 case for which it was 5 months were discarded, leaving 130 patients in our analysis. Due to missing data the number of cases analysed for some individual parameters was lower. 


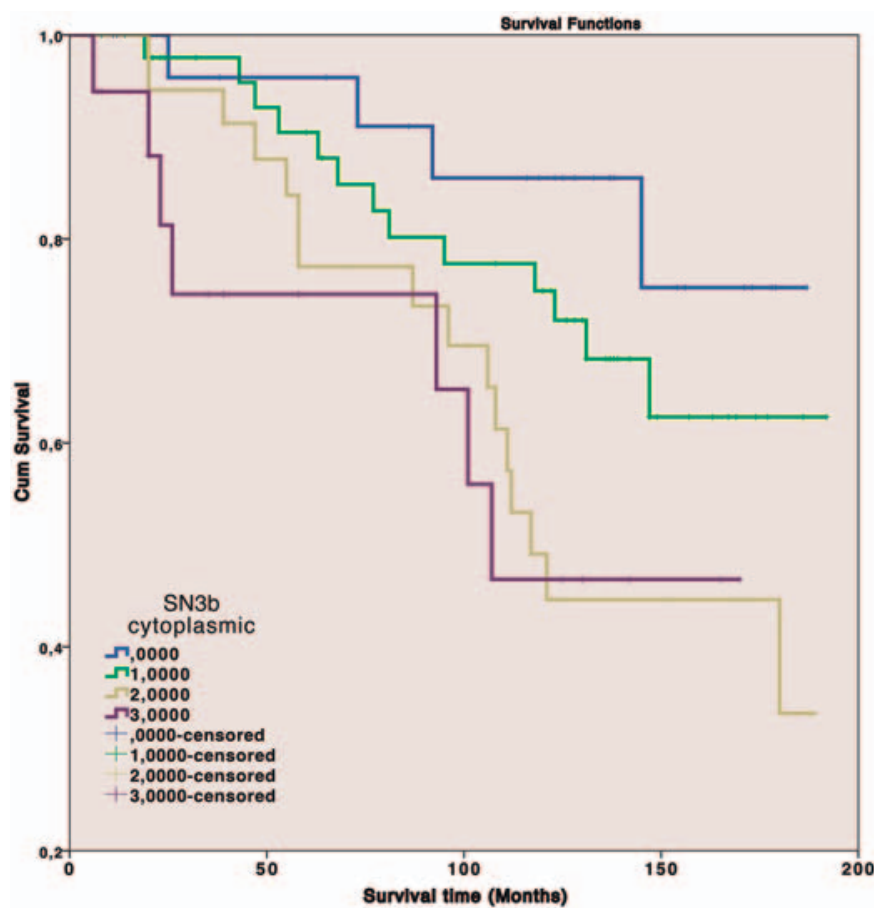

Fig. 1. Survival times from the Joint Cancer Register of the federal states of Berlin, Brandenburg, Mecklenburg-West Pomerania, Saxony-Anhalt, the free states of Saxony and Thuringia (GKR) and from the Berlin resident registration office.

We found a significant association of CD24 expression with a positive nodal status (table 1). This association between $\mathrm{pN}$ and cytoplasmic SN3b was further tested using the gamma statistics, which is suitable for analysing associations between ordinal-scaled variables. The corresponding cross-table (table 1) showed a positive association between cytoplasmic SN3b and pN. A gamma test for no association between cytoplasmic SN3b and $\mathrm{pN}$ (gamma test) was rejected with a significance level of $\mathrm{p}=0.013$.

\section{Survival Analyses}

We conducted a univariate Cox regression to determine the prognostic value for overall survival of CD24 and the standard prognostic parameters (pT, pN, G and oestrogen receptor) (table 2). The prognostic significance of CD24/SN3b was further confirmed in a Kaplan Meier analysis (fig. 1; p = 0.023 (log rank test)).

To approach a multivariate setting, backward stepwise (Wald) Cox regression analysis using the above prognostic parameters was employed: of the 130 cases, 15 were omitted due to missing data, leaving 115 cases and 34 events. In the resulting model, cytoplasmic CD24/SN3b immunoreactivity was the strongest independent prognostic parameter (table 3 ).

Despite the small number of cases, we considered it of interest to examine total survival time as a function of cytoplasmic SN3b for pN0 cases only (table 4). As with the above calculations, 60 cases (64 minus 2 cases censored before the earliest event in the stratum and 2 cases with missing values) with 12 deaths were included in the calculation, which yielded a significance of $\mathrm{p}=0.045$
Table 3. Multivariate Cox regression of CD24 expression and clinicopathological parameters - overall survival

\begin{tabular}{llll}
\hline & & Sig. & $\operatorname{Exp}(\mathrm{B})$ \\
\hline Step 1 & pT & 0.171 & 1.340 \\
& pN & 0.134 & 1.642 \\
& G & 0.061 & 1.625 \\
& ER & 0.895 & 0.952 \\
& CD24/SN3bc & 0.007 & 1.688 \\
Step 2 & pT & 0.172 & 1.334 \\
& pN & 0.120 & 1.656 \\
& G & 0.041 & 1.644 \\
& CD24/SN3bc & 0.007 & 1.691 \\
& pN & 0.035 & 1.866 \\
& G & 0.051 & 1.621 \\
& CD24/SN3bc & 0.007 & 1.693 \\
\hline
\end{tabular}

Table 4. Multivariate Cox regression of CD24/SN3b expression and clinicopathological parameters ( $\mathrm{pN} 0)$ overall surviva

\begin{tabular}{llll}
\hline & & Sig. & $\operatorname{Exp}(\mathrm{B})$ \\
\hline Step 1 & pT & 0.170 & 4.728 \\
& G & 0.827 & 0.888 \\
& ER & 0.108 & 0.338 \\
& SN3b-cytoplasmic & 0.031 & 1.899 \\
Step 2 & pt & 0.171 & 4.678 \\
& ER & 0.091 & 0.361 \\
& SN3b-cytoplasmic & 0.032 & 1.894 \\
Step 3 & ER & 0.067 & 0.338 \\
& SN3b-cytoplasmic & 0.045 & 1.779 \\
\hline
\end{tabular}

(backwards stepwise Cox regression). The same calculation for all pT1pN0 cases shows that the number of cases remains too small (data not shown).

\section{Discussion}

Our reanalysis revealed that cytoplasmic CD24/SN3b immunoreactivity is found to improve the prognostic accuracy further in terms of breast cancer-specific survival, even compared to nodal status. We are well aware that calculating breast cancer-specific survival is controversial due to diagnostic uncertainty in death statistics - as a result, for example, of death certificates not always being filled out correctly [4-6] or of treatment-induced premature deaths not directly caused by breast cancer. For this reason we deliberately used overall patient survival times. The results for the pN0 cases appear to support the validity of this method.

The increase in events detected using our data collection methodology of incorporating data from additional cancer registries is beneficial for this comparative analysis. Although the appropriateness of the Kaplan-Meier method can be questioned in cohorts with complex follow-up compositions like ours [3], we have still utilized this widely applied technique for approximation and the subsequent Cox analyses confirmed the prognostic value of CD24/ $\mathrm{SN} 3 \mathrm{~b}$. These results for cytoplasmic CD24/SN3b are even more interesting in light of recent data that led to discussions on the value of axillary lymph node dissection in cases in which several sentinel 
lymph nodes (up to 2) are positive [7-9]. This could imply that the quantity of lymph node involvement would no longer be available as a prognostic factor.

Unfortunately, the number of cases in this study is too low to identify putative pT1pN0 cases by an evaluation of cytoplasmic CD24/SN3b. If this were possible, it would identify a subgroup of small tumours with no lymph node involvement that still have a poor prognosis. In addition, currently the idea that local lymph node dissection is not significant for further survival is under considerable discussion [10].

Clearly, a much larger study of well-characterized breast cancer cases is not only warranted but also urgently needed to elucidate the promising prognostic value of SN3b immunoreactivity, ideally in direct comparison to an antibody that recognizes the CD24 protein core (e.g. SWA11). CD24 began to attract the attention of can- cer researchers a decade ago. It is overexpressed in the majority of human carcinomas and is often associated with a poorer prognosis [11]. Many of these studies were carried out with the SN3b antibody; therefore, it is time to investigate and annotate the SN3b epitope to understand its tumour-biological functions.

In conclusion, CD24/Ab-2 (clone SN3b) immunoreactivity is a strong prognostic factor in node-negative primary breast carcinomas and provides useful information supplementing that obtained from lymph node involvement.

\section{Disclosure Statement}

We declare that we have no conflict of interest.

\section{References}

1 Kristiansen G, Winzer KJ, Mayordomo E, et al.: CD24 expression is a new prognostic marker in breast cancer. Clin Cancer Res 2003;9:4906-4913.

2 Kristiansen G, Machado E, Bretz N, et al.: Molecular and clinical dissection of CD24 antibody specificity by a comprehensive comparative analysis. Lab Invest 2010;90:1102-1116.

3 Winzer KJ, Bellach J: Wertigkeit der routinemäßig erfassten Nachsorgedaten bei Brustkrebspatientinnen. Zentralbl Chir 2010;135:257-261.

4 Modelmog D: Todesursachen sowie Häufigkeit pathologisch-anatomischer Befundkomplexe und Diagnosen einer mittelgroßen Stadt bei fast 100 prozentiger $\mathrm{Ob}$ duktionsquote - Eine Obduktionsanalyse unter Ein beziehung histologischer Untersuchungen zum gegenwärtigen Stellenwert der Pathologie (Görlitzer Studie 1986/ 1987). Egelsbach, Köln, New York: HänselHohenhausen; 1993.
5 Modelmog D, Rahlenbeck S, Trichopoulos D: Accuracy of death certificates: A population-based, complete-coverage, one-year autopsy study in East Germany. Cancer Causes Control 1992;3:541-546.

6 Jahn I, Jöckel K-H, Bocter N, Müller W: Studie zur Verbesserung der Validität und Reliabilität der amtlichen Todesursachenstatistik. Band 52, Schriftenreihe des Bundesministeriums für Gesundheit. Baden-Baden: Nomos Verlagsgesellschaft; 1995.

7 Giuliano AE, Hunt KK, Ballman KV, et al.: Axillary dissection vs no axillary dissection in women with invasive breast cancer and sentinel node metastasis: A randomized clinical trial. JAMA 2011;305:569-575
8 Giuliano AE, McCall L, Beitsch P, et al.: Locoregional recurrence after sentinel lymph node dissection with or without axillary dissection in patients with sentinel lymph node metastases: The American College of Surgeons Oncology Group Z0011 randomized trial. Ann Surg 2010;252:426-432.

9 Haffty BG, Hunt KK, Harris JR, Buchholz TA: Positive sentinel nodes without axillary dissection: Implications for the radiation oncologist. J Clin Oncol 2011;29: 4479-4481.

10 Engel J, Emeny RT, Hölzel D: Positive lymph nodes do not metastasize. Cancer Metastasis Rev 2012;31:235246.

11 Kristiansen G, Sammar M, Altevogt P: Tumour biological aspects of $\mathrm{CD} 24$, a mucin-like adhesion molecule. J Mol Histol 2004;35:255-262. 